\title{
Phase-contrast tomography at the nanoscale using hard $x$ rays
}

\author{
Marco Stampanoni, ${ }^{1,2}$ Rajmund Mokso, ${ }^{1}$ Federica Marone, ${ }^{1}$ Joan Vila-Comamala, ${ }_{1}$ Sergey Gorelick, ${ }^{1}$ Pavel Trtik, ${ }^{3}$ \\ Konstantin Jefimovs, ${ }^{3}$ and Christian David ${ }^{1}$ \\ ${ }^{1}$ Paul Scherrer Institut, 5232 Villigen, Switzerland \\ ${ }^{2}$ Institute for Biomedical Engineering, University of Zürich and ETH Zürich, 8092 Zürich, Switzerland \\ ${ }^{3}$ Materials Science and Technology, EMPA, 8600 Dübendorf, Switzerland
}

(Received 25 February 2010; published 20 April 2010)

\begin{abstract}
Synchrotron-based full-field tomographic microscopy established itself as a tool for noninvasive investigations. Many beamlines worldwide routinely achieve micrometer spatial resolution while the isotropic 100-nm barrier is reached and trespassed only by few instruments, mainly in the soft x-ray regime. We present an x-ray, full-field microscope with tomographic capabilities operating at $10 \mathrm{keV}$ and with an isotropic resolution of 144 $\mathrm{nm}$. Custom-designed optical components allow for ideal, aperture-matched sample illumination and very sensitive phase contrast imaging. We show here that the instrument has been successfully used for the nondestructive, volumetric investigation of single cells.
\end{abstract}

DOI: 10.1103/PhysRevB.81.140105

PACS number(s): 68.37.Yz, 87.64.mh, 87.59.-e, 07.85.Tt

The high-resolution, large penetration depth, and chemical speciation of synchrotron-based tomography provides volumetric information of millimeter-sized specimens with voxel sizes in the micrometer range. ${ }^{1}$ Most of the end stations installed worldwide are based on parallel-beam geometry, the micrometer resolution limit being then essentially given by the spatial resolution of the detector. To reach and trespass the isotropic barrier of $100 \mathrm{~nm}$ however, the use of magnifying $\mathrm{x}$-ray optics is mandatory. This challenge has been tackled efficiently only by few instruments worldwide, mostly in the soft $\mathrm{x}$-ray range. ${ }^{2,3}$ When imaging biological samples, $\mathrm{x}$-rays between 283 and $530 \mathrm{eV}$ (the so-called water window) generate high-contrast images because of the large photoelectric cross-section difference between the carbonbased structures and the surrounding aqueous medium. However, the short focal length of Fresnel zone plates (FZPs) (used as microscope optics) within this energy range combined with the inherent strong absorption of air along the beam path, results in complex instrumental equipment and unpractical experimental conditions, especially when the sample needs to be cryocooled in order to reduce radiation damage. Moreover, the usage of soft $\mathrm{x}$-ray microscopes is problematic for thick specimens, as the penetration capability is low, and the small depth of focus limits the resolution. Operating a microscope at hard $\mathrm{x}$-rays could solve part of these problems since the higher penetration depth of the radiation, together with the larger depth-of-focus of the optical components, would allow for the investigation of thicker samples relaxing the experimental conditions. At hard x-ray energies, however, biological samples-consisting mainly of low- $Z$ elements-exhibit a small photoelectric cross section, resulting in a poor absorption signal and making the use of phase contrast unavoidable. In this work we introduce an instrument, which efficiently records tomographic absorption and phase contrast images at measured three-dimensional (3D) spatial resolution of $144 \mathrm{~nm}$.

One of the most common approaches to efficiently achieve sub-100-nm resolution is full-field microscopy. For this configuration, the key optical elements are a condenser, to provide illumination of the sample, and an objective lens, to produce a magnified image of the sample on the detector.
The illumination should be as homogeneous and as intense as possible and its numerical aperture should be matched to that of the objective lens in order to obtain optimum resolution. However, if the numerical aperture of the objective lens is larger than that of the condenser it is possible to collect stronger diffracting signals from the sample increasing the sensitivity of the system. ${ }^{4}$ Condensing x-rays with FZPs,${ }^{5}$ tapered capillaries, ${ }^{6}$ mirrors, ${ }^{7}$ or combinations of these devices ${ }^{8}$ is a common solution. These optics focus the beam into a Gaussian-shaped spot, normally smaller than the field of view of the microscope, requiring therefore the condenser to be shaken along the direction transverse to the beam to partially overcome this inhomogeneous illumination..$^{9}$ Beam shapers with complex functionality in the x-ray regime, as introduced originally by Di Fabrizio et $a l .{ }^{8}$ are usually difficult to realize since many unknown parameters of the beam have to be defined in advance and there are some restrictions resulting from the fabrication limitations. A simple beam-shaper design was recently suggested and tested with a soft x-ray full-field microscope ${ }^{10}$ The underlying idea was to divide a conventional FZP into sectors, keeping the local spatial frequency within each sector constant. At the beamline for Tomographic Microscopy and Coherent Radiology Experiments (TOMCAT) of the Swiss Light Source, ${ }^{11}$ we installed such a beam shaper fabricated according to an optimized design. ${ }^{12}$ Each subfield consists of a linear grating with constant line orientation and period both corresponding to the local orientation and period of the structures of the FZP. As a result, our beam shaper has the same focal length as the FZP, with the first diffraction orders of every subgrating forming exactly coinciding flat-top square illuminations in the focal plane of the device with the illuminated area equal to the size of the subgratings. Because each of them is equally contributing to the illumination, this device is almost insensitive to beam fluctuations and misalignments. Our beam shaper is $1 \times 1 \mathrm{~mm}^{2}$ in size, with subfields of $50 \times 50 \mu \mathrm{m}^{2}$ and $1-\mu \mathrm{m}$-thick gold structures. ${ }^{13}$ The outermost zone width is $\Delta r_{\mathrm{bs}}=100 \mathrm{~nm}$. At $10 \mathrm{keV}$ this yields a focal length of $f_{\mathrm{bs}}=1083 \mathrm{~mm}$ and, for a source located 20 $\mathrm{m}$ upstream, this results in a position of the illumination plane at $q_{\mathrm{bs}}=1145 \mathrm{~mm}$, see Fig. 1 . To block the zeroth order 


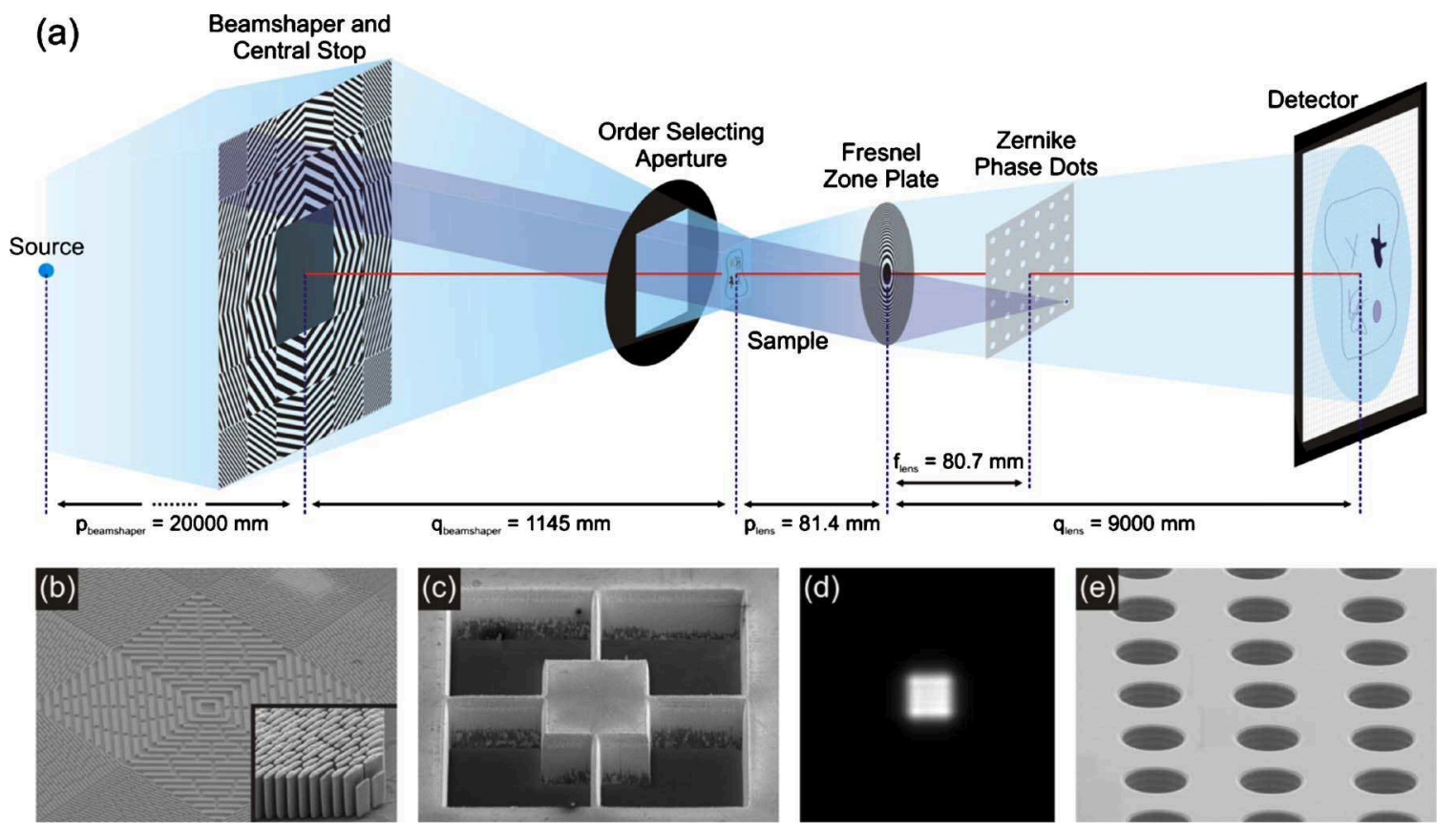

FIG. 1. (Color online) (a) Schematic of the nanoscope setup installed at the TOMCAT beamline. The beam shaper (b) collects light coming from the source (located $20 \mathrm{~m}$ upstream) and produces a top-flat square illumination (d) at distance $q_{\text {beamshaper }}$ The Fresnel zone plate magnifies this region into the detector located at $q_{\text {lens }}=9 \mathrm{~m}$ downstream. At the back-focal distance of the FZP the dot array (e) is aligned to the diffraction spots, generating Zernike phase contrast. An SEM image of the central part of the beam shaper is shown in (b) and the inset depicts the corner region, showing the $1-\mu \mathrm{m}$-thick gold structures $100 \mathrm{~nm}$ apart. The central stop, obtained by laser cutting a $250-\mu \mathrm{m}$-thick platinum/iridium foil is shown in (c).

through the condenser an opaque square central stop (600 $\times 600 \mu \mathrm{m}^{2}$ ) was cut out from $250-\mu \mathrm{m}$-thick platinum/ iridium foil by picosecond laser ablation. The size of the shadow formed by the central stop is matched to the size of the beam shaper illumination magnified by the objective lens on the detector. As objective lens, we used a gold FZP, obtained by electroplating into a polyimide mold, ${ }^{14}$ with an outermost zone width of $\Delta r=100 \mathrm{~nm}$, a zone height of 1 micron, and a diameter of $100 \mu \mathrm{m}$. The lens has a focal length of $f_{\text {lens }}=80.65 \mathrm{~mm}$ and, for a distance between lens and detector of $q_{\text {lens }}=9000 \mathrm{~mm}$, this results in a magnification factor of $M=110 \times$, see Fig. 1. The Rayleigh criterion predicts a best-case achievable resolution around $R_{\text {Rayleigh }}$ $=1.22 \cdot \Delta r=122 \mathrm{~nm}$. Each sector of the beam shaper produces a spot in the back-focal (BF) plane of the objective lens. The distance $d_{\text {spots }}$ between these spots (both in vertical and horizontal direction) can be obtained by geometrical arguments using the classical lens equation. If we consider two rays going through the centers of two adjacent square sectors of the beam shaper, it is obvious that they cross the plane of the Fresnel-zone plate with a separation of $d_{\text {rays }}=\operatorname{size}_{\text {sector }} \cdot p_{\text {lens }} / q_{\text {bs }}=3.5537$ microns. After passing through the lens, the rays will point to the center of the detector: their separation and therefore the distance between the spots in the BF plane of the lens will be slightly smaller and is given by $d_{\text {spots,BF }}=d_{\text {rays }} \cdot\left(q_{\text {lens }}-f_{\text {lens }}\right) / q_{\text {lens }}=3.5218$ microns. Further the beams through each sector of the beam shaper-being generated from the source situated at $p_{\mathrm{bs}}+q_{\mathrm{bs}}+p_{\text {lens }}$ upstream of the objective lens-will not be exactly focused in the back-focal plane of the lens but at a slightly larger distance $a=\frac{\left(p_{B S}+q_{B S}+p_{\text {lenn }}\right) \cdot f_{\text {lens }}}{\left(p_{B S}+q_{B S}+p_{\text {lens }}\right)-f_{\text {lens }}}$ downstream from the zone plate. The true value of the spot distance is given finally by: $d_{\text {spots }}=\operatorname{size}_{\text {sector }} \cdot \frac{\left(q_{\text {lens }} \cdot f_{\text {lens }}\right)}{\left(q_{\text {lens }}-f_{\text {lens }}\right)} \cdot \frac{\left(p_{b s}-f_{b s}\right)}{\left(p_{b s} \cdot f_{b s}\right)} \cdot \frac{q_{\text {lens }} a}{q_{\text {lens }}}$. Since $f \approx a$, this correction is very small: in fact the final value for the distance between the spots at distance $a$ downstream to zone plate is $d_{\text {spots }}=3.5217$ microns. A variety of phasesensitive techniques have been developed for full-field X-ray microscopes including both Zernike ${ }^{15,16}$ and differential ${ }^{17,18}$ phase contrast imaging methods. Zernike phase contrast microscopes have traditionally been implemented using hollow cone illumination and an imaging objective in combination with a separate $\pm \pi / 2$ phase ring. The dimensions of the phase ring have to match the geometry of the undiffracted light from the sample and the ring is placed in the back-focal plane of the imaging objective. Contrast then arises from the fact that the phase between the diffracted wave front and the nondiffracted one is shifted. ${ }^{19}$ In our case, due to the particular illumination geometry (an array of spots) at the backfocal length $f_{\text {lens }}$ the phase ring will no longer work and it must be replaced by a phase dot array, see Fig. 1. The depth of the dots (etched into silicon) is $\operatorname{dot}_{\text {depth }}=6.34 \mu \mathrm{m}$, corresponding to a phase shift of $\pi / 2$ at $10 \mathrm{keV}$. The use of phase dots instead of more conventional rings is imposed by the illumination geometry but it brings an important advantage in terms of image quality. Using such discrete structures as phase shifting elements reduces the "halo" artifacts. ${ }^{20}$ In Zernike phase contrast, the halo arises when the diffracted beam passes through the ring in such a manner that its phase is shifted by the same amount as that of the direct, undiffracted beam. With our system, where phase shifting is obtained by discrete structures, the halo effect can be regulated by the size of individual phase holes. Careful optimization of 
the current setup resulted in images where also large structures (usually the most affected by the halo artifacts) could be readily interpreted.

As a detector we used a Photonic Science VHR Image Star X-ray camera based on a full-frame transfer Kodak charge coupled device $(C C D)$ with $3056 \times 3056$ pixels of $12 \times 12 \mu \mathrm{m}^{2}$ size. It features a full well capacity larger than $110 \mathrm{ke}^{-} /$pixel with a readout noise at $8 \mathrm{MHz}$ of less than ten electrons and dark current smaller than 0.5 electrons/pixel/ second. The chip size is matched via a 1:3 magnifying fiber optic taper (FOP). A $2.5 \mathrm{mg} / \mathrm{cm}^{2} \mathrm{GdOS}: \mathrm{Tb}$ is deposited on the FOP and the final assembly results in an active input window of $12 \times 12 \mathrm{~mm}^{2}$ with an optical resolution of $4 \mu \mathrm{m}$. For $M=110 \times$ this yields a theoretical pixel size of $36 \times 36 \mathrm{~nm}^{2}$

The mechanical stability of the system has been investigated in a previous work, where the nanoscope has been operated in absorption mode, i.e, without phase dot array. ${ }^{21}$ By cross-correlating time-resolved $\mathrm{x}$-ray nanoradiographic projections of a high-resolution gold mesh (Plano G2786A), we observed horizontal and vertical drifts within a range of $\pm 100 \mathrm{~nm}$ during one hour, which is by far longer than what is needed for a complete tomographic scan. Namely, at 10 $\mathrm{keV}$, the 14 bit CCD detector is almost saturated with an exposure time of $1 \mathrm{~s}$ and a tomographic scan of 251 projections can be acquired within a few minutes. For the results presented here, we operated the nanoscope in Zernike phase contrast mode, i.e., we carefully positioned the phase dot array in the back-focal plane of the objective lens using two crossmounted piezo stages and a motorized rotational stage. In a first experiment, we investigated the resolution and sensitivity of the system by imaging two different phase objects. Figure 2(a) shows a radiographic projection of a silicon $\mathrm{Si}$ emens star: the structures of $1-\mu \mathrm{m}$ depth induce a phase shift of $\pi / 15$ at $10 \mathrm{keV}$. An area of $350 \times 350 \mu \mathrm{m}^{2}$ has been covered by stitching $10 \times 10$ single images each of them accommodating a $35 \times 35 \mu \mathrm{m}^{2}$ field of view and pixel size of $36 \times 36 \mathrm{~nm}^{2}$. Due to the high efficiency of the system, the whole area could be sampled in less than 2 min. Figure 2(b) shows a closer look of the central part of the silicon Siemens star. This region obviously contains high-frequency components and has been used to estimate the two-dimensional (2D) spatial resolution of the nanoscope using a powerspectrum-based approach. ${ }^{22}$ The calculations return a true resolution around $R_{\text {true }}=133 \mathrm{~nm}$, i.e approx. $3.7 \times$ the theoretical pixel size. This value is very close to the limit of $R_{\text {Rayleigh }}=122 \mathrm{~nm}$ confirming that the system is performing almost at its diffraction limit. As a comparison, Fig. 2(c) illustrates the same central region obtained by scanning electron microscopy.

Figure 2(d) shows a radiographic projection of a $30 \mu \mathrm{m}$ diameter $\mathrm{SiO}_{2}$ sphere (Microspheres-Nanospheres C-S1030.0) with some spheroidal inclusions. We tomographically investigated it by acquiring 721 equiangular projections between 0 and $180^{\circ}$. Figures $2(\mathrm{e})$ and 2(f) show axial and sagittal cuts through the sample, clearly revealing the internal structure. We determined the (3D) spatial resolution using the same power-spectrum method ${ }^{22}$ and we obtained a value of $R_{\text {true }}=144 \mathrm{~nm}$.

The overall aim of our work is to develop an instrument

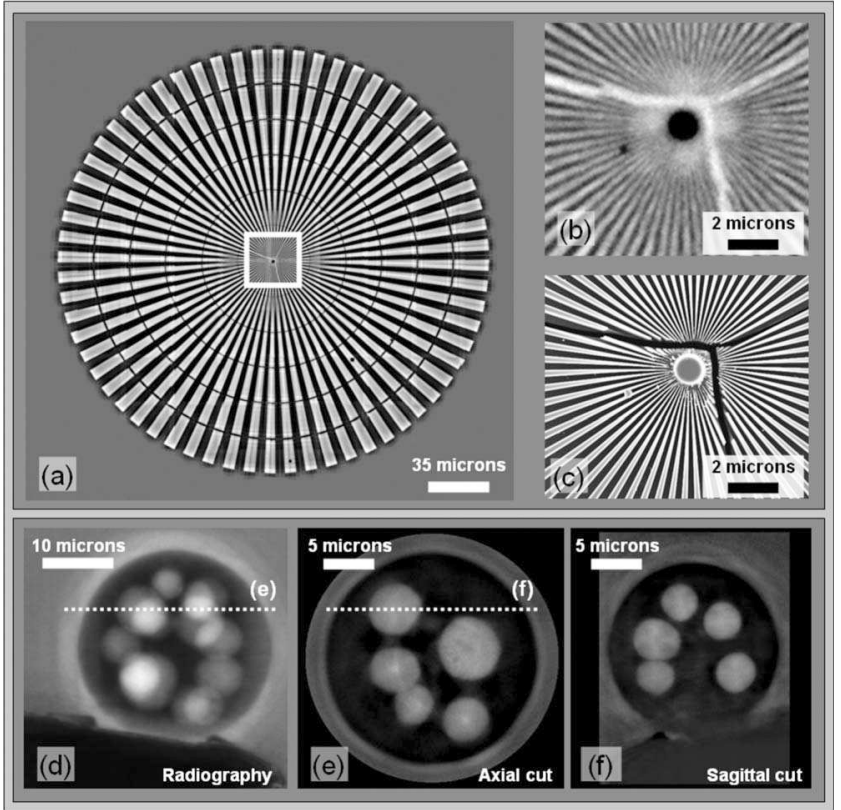

FIG. 2. Zernike phase contrast nanoimaging of different calibration samples. [(a)-(c)]: Radiographic image of a silicon Siemens star. (a) shows an image obtained by stitching $10 \times 10$ single snapshots with a field of view of $35 \times 35$ microns each. (b) illustrates the central part of the star and (c) illustrates the same region imaged with SEM. [(d)-(f)]: Nanotomographic investigation of a $\mathrm{SiO}_{2}$ sphere with inclusions. (d) depicts a nanoradiographic projection. The dotted line marks the height of the axial cut shown in (e). (f) shows a sagittal cut through the sample at the position marked in (e).

which can visualize soft tissue at high resolution without the need of any staining techniques. Coupled with the large penetrating power of hard x-rays such an instrument would be extremely useful when tomographic imaging of small, poorly absorbing samples such as cells becomes an issue.

In a second experiment, we imaged MC3 preosteoblast cells-fixed but not stained-contained in a glass capillary (100 $\mu \mathrm{m}$ diameter, $10 \mu \mathrm{m}$ wall thickness) and suspended in a $1.3 \mathrm{wt} \%$ hydrogel. The measurement has been done at 10

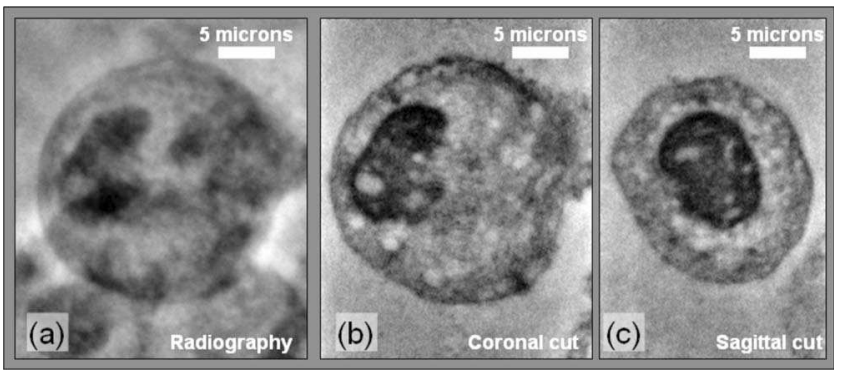

FIG. 3. Zernike phase contrast nanotomography of an unstained MC3 preosteoblast cell. (a) shows a nanoradiographic projection of the MC3 cell together with other cells which happened to be on the beam path during the snapshot. (b) and (c) show coronal and sagittal cut of the MC3 cell, respectively. A total of 251 angular projections over $180^{\circ}$ have been collected within $10 \mathrm{~min}$. Clearly visible are the nucleus (dark) with several intracellular structures. 
$\mathrm{keV}$, in Zernike phase contrast mode. Figure 3(a) shows a radiographic projection of a MC3 cell obtained at a pixel size of $72 \times 72 \mathrm{~nm}^{2}$. Other cells are visible (in the bottom left corner for instance), since the radiographic approach, of course, squeezes a 3D structure onto a $2 \mathrm{D}$ image. At this point the advantages of a hard x-ray microscope become obvious: the investigated cell can be aligned in such a way that during a $\left[0,180^{\circ}\right]$ rotation it stays within the field of view of the microscope. Other cells or the capillary itself, which might appear on the beam path during rotation, can be simply neglected. From the viewpoint of the tomographic proto$\mathrm{col}$, this means that we are acquiring information of the cell following a local tomography approach: this will affect the quantitative interpretation of the data (since the gray values distribution will be different from slice to slice) but will return the morphological information correctly. Figures 3(b) and $3(\mathrm{c})$ in fact, show coronal and sagittal slices through the MC3 cell obtained with a tomographic scan consisting of 251 projections acquired in approximately $10 \mathrm{~min}$. The orthogonal slices depicted in Figs. 3(b) and 3(c) contain additional (volumetric) information about the cell internal structure that would be impossible to obtain with conventional 2D approaches and without using staining methods.

In this work we presented a full-field microscope which operates with broadband hard $\mathrm{x}$-rays. We used a $[\mathrm{Ru} / \mathrm{C}]_{100}$ double-bounce multilayer monochromator providing an energy bandwidth of $2 \%$ at $10 \mathrm{keV}{ }^{11}$ The fact that the microscope, when operated in full-field mode, almost reached its theoretical limits (133 nm against the Rayleigh limit of 122 $\mathrm{nm}$ ) shows that the system performs excellently also with broadband radiation. We have introduced an alternative optical design which improves the quality of the illumination, making it ideally suited for classical, absorption-based microscopic applications. The system can also be operated in phase-contrast mode by positioning a novel optical component in the back-focal plane of the objective lens. We demonstrated that this configuration can be efficiently used either for simple radiographic investigations or even for nanotomographic experiments of a variety of materials. The large penetration depth of the hard x-ray probe in combination with local tomography methods, successfully allows the volumetric (3D) study of large samples. Finally, the use of hard $\mathrm{x}$-rays in phase contrast mode contributes to reduce dose deposition to sensitive samples.

We greatly acknowledge G. Mikuljan of the SLSTOMCAT beamline staff for his fundamental support in setting up the experiment. We thank J. Vörös and A. Sala Roca from the Institute of Biomedical Engineering of the University and ETH Zürich for the preparation of the MC3 preosteoblast cells. P. Modregger of the SLS-TOMCAT team also deserves our thanks for the estimation of the true resolution with the power spectrum method. We are also indebted to $\mathrm{M}$. Feser of Xradia Inc. for valuable discussions on Zernike phase contrast.
${ }^{1}$ U. Bonse, Proc. SPIE 7078, 707802 (2008).

${ }^{2}$ D. Weiss, G. Schneider, B. Niemann, P. Guttmann, D. Rudolph, and G. Schmahl, Ultramicroscopy 84, 185 (2000).

${ }^{3}$ W. Meyer-Ilse, D. Hamamoto, A. Nair, S. A. Lelievre, G. Denbeaux, L. Johnson, A. L. Pearson, D. Yager, M. A. Legros, and C. A. Larabell, J. Microsc. 201, 395 (2001).

${ }^{4}$ M. Born and E. Wolf, Principles of Optics, 7th ed. (Cambridge University Press, Cambridge, 1999), p. 595.

${ }^{5}$ E. H. Anderson, D. L. Olynick, B. Harteneck, E. Veklwrov, G Denbeaux., W. L. Chao, A. Lucero, L. Johnson, and D. Attwood, J. Vac. Sci. Technol. B 18, 2970 (2000).

${ }^{6}$ G. C. Yin, Y. F. Song, M. T. Tang, F. R. Chen, K. S. Liang, F. W. Duewer, M. Feser, W. Yun, and H. P. D. Shieh, Appl. Phys. Lett. 89, 221122 (2006).

${ }^{7}$ C. Rau, V. Crecea, K. M. Peterson, P. R. Jemian, C. P. Richter, U. Neuhäusler, G. Schneider, X. Yu, P. V. Braun, and I. K. Robinson, Proceedings of the 8th International Conference: X-ray Microscopy, IPAP Conference Series Vol. 7 (Himenji, Japan, 2005), pp. 7-8.

${ }^{8}$ E. Di Fabrizio, S. Cabrini, D. Cojoc, F. Romanato, L. Businaro, M. Altissimo, B. Kaulich, T. Wilhein, J. Susini, M. De Vittorio, E. Vitale, G. Gigli, and R. Cingolani, Microelectron. Eng. 6768, 87 (2003).

${ }^{9}$ B. Niemann, P. Guttmann, S. Rehbein, and C. Knöchel, J. Phys. IV 104, 273 (2003).

${ }^{10}$ U. Vogt, M. Lindblom, P. Charalambous, B. Kaulich, and T.
Wilhein, Opt. Lett. 31, 1465 (2006).

${ }^{11}$ M. Stampanoni, A. Groso, A. Isenegger, G. Mikuljan, Q. Chen, A. Bertrand, S. Henein, R. Betemps, U. Frommherz, P. Böhler, D. Meister, M. Lange, and R. Abela, Proc. SPIE 6318, 63180M (2006).

${ }^{12}$ K. Jefimovs, J. Vila-Comamala, M. Stampanoni, B. Kaulich, and C. David, J. Synchrotron Radiat. 15, 106 (2008).

${ }^{13}$ S. Gorelick, J. Vila-Comamala, V. Guzenko, M. Stampanoni, R. Mokso, and C. David, Microelectron. Eng., 87, 1052 (2010).

${ }^{14}$ K. Jefimovs, O. Bunk, F. Pfeiffer, D. Grolimund, J. F. van der Veen, and C. David, Microelectron. Eng. 84, 1467 (2007).

${ }^{15}$ U. Neuhäusler, G. Schneider, W. Ludwig, M. A. Meyer, E. Zschech, and D. Hambach, J. Phys. D 36, A79 (2003).

${ }^{16}$ H. S. Youn and S. W. Jung, J. Microsc. 223, 53 (2006).

${ }^{17}$ U. Vogt, M. Lindblom, P. A. C. Jansson, T. T. Tuohimaa, A. Holmberg, H. M. Hertz, M. Wieland, and T. Wilhein, Opt. Lett. 30, 2167 (2005).

${ }^{18}$ A. Sakdinawat and Y. Liu, Opt. Lett. 32, 2635 (2007).

${ }^{19}$ F. Zernike, Physica 1, 689 (1934).

${ }^{20} \mathrm{C}$. Maurer, A. Jesacher, S. Bernet, and M. Ritsch-Marte, Opt. Express 16, 19821 (2008).

${ }^{21}$ M. Stampanoni, F. Marone, G. Mikuljan, K. Jefimovs, P. Trtik, J. Vila-Comamala, C. David, and R. Abela, J. Phys.: Conf. Ser. 186, 012018 (2009).

${ }^{22}$ P. Modregger, D. Lübbert, P. Schäfer, and R. Köhler, Phys. Status Solidi A 204, 2746 (2007). 